\title{
Estimation of Initial Conditions and Secure Communication
}

\author{
Anil Maybhatd 11,2 , R.E.Amritkar ${ }^{1}$ and D. R. Kulkarnit 1 . \\ ${ }^{1}$ Physical Research Laboratory, Navrangpura, Ahmedabad 380009, India \\ ${ }^{2}$ Department of Physics, University of Pune, Pune 41100\%, India
}

\begin{abstract}
We estimate the initial conditions of a multivariable dynamical system from a scalar signal, using a modified Newton-Raphson method incorporating the time evolution. We can estimate initial conditions of periodic and chaotic systems and the required length of scalar signal is very small. We also find that the information flow from one variable to the other has logarithmic dependence on time. An important application of the method is in secure communications. The communication procedure has several advantages as compared to others using dynamical systems.
\end{abstract}

PACS number(s): 05.45.-a, 05.45.Vx, 05.45.Tp, 05.45.Xt

A trajectory of a given dynamical system in its state space depends on the set of initial conditions with which it starts. In particular, the state of a chaotic system at a later time is exponentially sensitive to changes in its initial state [1]. This feature of a chaotic system leads to a complex behaviour in state space that appears random yet is deterministic, and the time evolution is uniquely fixed by the initial state of the system. Though there are several invariant measures of a chaotic system which are not sensitive to the initial conditions, the exact trajectory crucially depends on the initial state and hence is difficult to reproduce due to sensitivity to initial conditions.

In light of these facts, it is interesting and important to ask whether the complete set of initial conditions of a given multivariable dynamical system can be estimated from a given scalar time series of a single state space variable. In this letter we present a novel and simple method to estimate the initial conditions from a given scalar time series. The method is based on a modified multidimensional Newton-Raphson method [1,2] that includes the time evolution of the system. The length of the time series required for estimating the initial conditions is very small. Also, the method works even when the conditional Lyapunov exponents are positive.

An important application of our method is in the area of secure communications 3,4]. A nice feature of this application is that the signal that is transmitted is not the one modulated by the information signal, making it difficult to crack the method. Our method is also useful in the problem of synchronization of chaotic signals [5]. The knowledge of initial conditions means the response system can be be synchronized with the drive system almost instantaneously thereby removing the problem of transients. Also synchronization can be achieved in most of the cases where other methods fail [5].
Let us consider an autonomous dynamical system given by,

$$
\dot{\mathbf{x}}=\mathbf{F}(\mathbf{x})
$$

where $\mathbf{x}=\left(x_{1}, x_{2}, \ldots, x_{d}\right)$ is a $d$-dimensional state vector whose evolution is governed by the function $\mathbf{F}=$ $\left(F_{1}, F_{2}, \ldots, F_{d}\right)$. Given an initial state vector $\mathbf{x}(0)$ at time $t=0$, the time evolution $\mathbf{x}(t)$ is uniquely determined by Eq. (価). Now let us assume that only one component of the state vector is known to us and we take it to be $x_{1}(t)$ without loss of generality. The problem that we address is to obtain the initial state vector $\mathbf{x}(0)$ from the knowledge of the scalar signal $x_{1}(t)$.

Let $\mathbf{y}(0)$ denote a random initial state vector and $\mathbf{y}(t)$ its time evolution obtained from Eq. (11). Let $\mathbf{w}(t)$ denote the difference $\mathbf{w}(t)=\mathbf{y}(t)-\mathbf{x}(t)$. We look for the solution of the equation

$$
\mathbf{w}(t)=0 .
$$

Noting that the initial state vectors $\mathbf{y}(0)$ and $\mathbf{x}(0)$ uniquely determine the difference $\mathbf{w}(t)$, one of the solutions of Eq. (2) is $\mathbf{y}(\mathbf{0})-\mathbf{x}(0)=0$ and this is the solution that we are searching for.

We now introduce the notation $\mathbf{w}^{n}=\mathbf{w}^{n}\left(\mathbf{y}^{0}, \mathbf{x}^{0}\right)=$ $\mathbf{w}(n \Delta t)$, where $\Delta t$ is a small time interval. Similarly, $\mathbf{y}^{n}=\mathbf{y}(n \Delta t)$ and $\mathbf{x}^{n}=\mathbf{x}(n \Delta t)$. With this notation condition (2) can be written as $\mathbf{w}^{n}=0$.

Our approach to the solution of Eq. (2) is a modified Newton-Raphson method [1] which includes the time evolution of the system.

Let us first consider $\mathbf{w}^{1}$. We have

$$
\begin{aligned}
0 & =\mathbf{w}^{1}\left(\mathbf{x}^{0}, \mathbf{x}^{0}\right)=\mathbf{w}^{1}\left(\mathbf{y}^{0}+\delta \mathbf{y}^{0}, \mathbf{x}^{0}\right), \\
& =\mathbf{w}^{1}\left(\mathbf{y}^{0}, \mathbf{x}^{0}\right)+\left(\delta \mathbf{y}^{0} \cdot \nabla_{\mathbf{y}^{\circ}}\right) \mathbf{w}^{1}\left(\mathbf{y}^{0}, \mathbf{x}^{0}\right)+\mathcal{O}\left(\left(\delta \mathbf{y}^{0}\right)^{2}\right),
\end{aligned}
$$

\footnotetext{
*e-mail: nil@prl.ernet.in

${ }^{\dagger}$ e-mail: amritkar@prl.ernet.in

${ }^{\ddagger}$ e-mail: drkul@prl.ernet.in
} 
where $\delta \mathbf{y}^{0}=\mathbf{x}^{0}-\mathbf{y}^{0}=-\mathbf{w}^{0}$ and the last step is a Taylor series expansion in $\delta \mathbf{y}^{0}$. For small $\Delta t$, we can write

$$
\mathbf{w}^{1}\left(\mathbf{y}^{0}, \mathbf{x}^{0}\right)=\mathbf{w}^{0}+\Delta t\left[\mathbf{F}\left(\mathbf{y}^{0}\right)-\mathbf{F}\left(\mathbf{x}^{0}\right)\right]+\mathcal{O}\left((\Delta t)^{2}\right) .
$$

Substituting Eq. (1) in Eq. (3) and neglecting higher order terms, we get,

$$
\mathbf{w}^{1}\left(\mathbf{y}^{0}, \mathbf{x}^{0}\right)=\mathbf{w}^{0}-\Delta t\left(\mathbf{w}^{0} \cdot \nabla_{\mathbf{y}^{\circ}}\right) \mathbf{F}\left(\mathbf{y}^{0}\right) .
$$

It is convenient to write the above equation in a matrix form as

$$
W^{1}=\left(I+\Delta t J^{0}\right) W^{0}=A^{0} W^{0},
$$

where $W^{n}$ is the column matrix corresponding to the vector $\mathbf{w}^{n}, I$ is the identity matrix, $A^{n}=I+\Delta t J^{n}$, and the elements of the Jacobian matrix $J^{n}$ are $J_{i j}^{n}=$ $\partial F_{i}\left(\mathbf{y}^{n}\right) / \partial y_{j}^{n}$.

Next we consider $\mathbf{w}^{2}$ or $W^{2}$. Proceeding as above, we get (see Eq. (6)),

$$
\begin{aligned}
W^{2} & =\left(I+\Delta t J^{1}\right) W^{1}=\left(I+\Delta t J^{1}\right)\left(I+\Delta t J^{0}\right) W^{0} \\
& =A^{1} A^{0} W^{0} .
\end{aligned}
$$

Similarly, the equation for $W^{n}$ is

$$
W^{n}=A^{n-1} A^{n-2} \cdots A^{0} W^{0} .
$$

We now concentrate on the first component of the signal whose time series is assumed to be known. For a $d$-dimensional system we need $d-1$ equations to determine the initial state vector $\mathbf{x}^{0}$. Eqs. (6), (7) and (8) give us the required relations.

$$
\begin{aligned}
W_{1}^{1} & =\sum_{i=1}^{d} A_{1 i}^{0} W_{i}^{0}, \\
W_{1}^{2} & =\sum_{i, j=1}^{d} A_{1 i}^{1} A_{i j}^{0} W_{j}^{0}, \\
\vdots & \\
W_{1}^{d-1} & =\sum_{i, \ldots, l, m=1}^{d} A_{1 i}^{d-2} \cdots A_{l m}^{0} W_{m}^{0},
\end{aligned}
$$

These are $d-1$ simultaneous equations for $W^{0}$.

The numerical procedure is as follows. We set the initial state of system (11) to a random initial guess vector $\left(\mathbf{y}^{0}\right)_{\text {old }}$ with $\left(y_{1}^{0}\right)_{\text {old }}=x_{1}^{0}$ and evolve it using Eq. (1). Using this vector $\mathbf{y}(t)$ we write down $d-1$ simultaneous equations (Eqs. (9)) which can be solved for $d-1$ unknown components of $\mathbf{w}^{0}=-\delta \mathbf{y}^{0}$. Also, $\delta y_{1}^{0}=0$. Thus the initial guess vector can be improved by

$$
\left(\mathbf{y}^{0}\right)_{\text {new }}=\left(\mathbf{y}^{0}\right)_{\text {old }}+\delta \mathbf{y}^{0} .
$$

This sets up an iterative scheme giving us better and better estimates of the initial vector which converge to $\mathrm{x}^{0}$.
We note that as in Newton-Raphson method, the choice of the initial guess vector can be very important [2]. In some cases, the iterative procedure of Eq. (10) may not converge or converge to a wrong root. In such cases, a different choice of initial guess vector can be useful.

We further note the similarity of our method with the so called method of variational equations in analytical dynamics [6]. The method of variational equations can be applied to a known Hamiltonian system to determine an unknown neighboring trajectory to an already known one. There, the method requires a complete particular solution of a known set of Hamiltonian equations of motion. In contrast, we have used our method for dissipative chaotic systems. In such systems an analytical solution of the equations of motion cannot be known. Further our method requires only one component of a complete trajectory to be sampled. This is important for the application of our method to secure communications as we will demonstrate afterwards.

We now illustrate our method of estimating the initial state. As an example we discuss the Rössler system given by [7], $\dot{\mathbf{x}}=\left(-x_{2}-x_{3}, x_{1}+a x_{2}, b+x_{3}\left(x_{1}-c\right)\right)$. First, we consider a case when the time series for $x_{1}$ is given and we want to estimate $\left(x_{2}^{0}, x_{3}^{0}\right)$. We chose the parameters $(a, b, c)$ such that the system is in the chaotic regime and the initial state $\mathbf{x}^{0}$ is on the chaotic attractor. We start with an arbitrary initial state $\mathbf{y}^{0}=\left(y_{1}^{0}, y_{2}^{0}, y_{3}^{0}\right)$ with $y_{1}^{0}=x_{1}^{0}$. From Eqs. (9) we get a pair of simultaneous equations for $\left(\delta y_{2}^{0}, \delta y_{3}^{0}\right)$, which can be solved to obtain these values. With $\delta y_{1}^{0}=0$ we use these in an iterative manner (Eq. (10)) to obtain the correct initial conditions.

Let $e_{i}=\left|y_{i}^{0}-x_{i}^{0}\right|$ denote the absolute error in the estimation of $x_{i}^{0}$. In Fig. 1(a) we plot a graph of errors $e_{2}$ and $e_{3}$ plotted against the number of iterations, $n$, of our method (Eq. (10)). From Fig. 1(a) we see that the errors go to zero and the successive estimates converge to the correct values of $\left(x_{2}^{0}, x_{3}^{0}\right)$. Using only two data points in the given time series $x_{1}(t)$, we can thus readily estimate the full initial state $\mathbf{x}^{0}$. We also note that the rate of convergence is very good. In about 8 to 10 iterates we obtain the initial values $\left(x_{2}^{0}, x_{3}^{0}\right)$ to within computer accuracy. If we write the deviations of the successive iterates from the correct values in the form

$$
\left(e_{i}\right)_{n}=\left|\left(y_{i}^{0}\right)_{n}-x_{i}^{0}\right| \sim e^{-\alpha n},
$$

where $n$ is the number of iterations, then the value of the parameter $\alpha$ is found to be 2.12 for $e_{2}$ and 2.10 for $e_{3}$. This is consistent with the fact that Newton-Raphson method has a quadratic convergence [1,2].

We note that the largest Lyapunov exponent for the subsystem $\left(y_{2}^{0}, y_{3}^{0}\right)$ (conditional or subsystem Lyapunov exponent) is positive [5]. The success of our method does 
not depend on whether this Lyapunov exponent is positive or negative. This is important for synchronization of chaotic signals.

We next present cases where time series for the variables $x_{2}$ and $x_{3}$ of the Rössler system are given. The procedure is similar to the case of time series for $x_{1}$ as discussed above. Fig. 1 (b) shows the errors $e_{1}$ and $e_{3}$, when time series for $x_{2}$ is given, plotted against the number of iterations. The parameter $\alpha$ (Eq. (11)) is 2.08 for $e_{1}$ and 2.10 for $e_{3}$. This again indicates a quadratic convergence. Similarly, Fig. 1 (c) shows the quantities $e_{1}$ and $e_{2}$ when time series for $x_{3}$ is given, as a function of the number of iterations. The parameter $\alpha$ (Eq. (11)) is 2.02 for $e_{1}$ and 2.10 for $e_{2}$. We note that the largest subsystem Lyapunov exponent is negative when time series for $x_{2}$ is given and is positive when time series for $x_{3}$ is given [5].

We have successfully applied our method to estimate the initial state vector using a given scalar time series for many other dynamical systems as well. These include Lorenz system [8] in its periodic, chaotic or intermittent regimes, Chua's circuit [9], the disk dynamo system modeling a periodic reversal of earth's magnetic field [10,11, a 3-d plasma system formed by a three wave resonant coupling equations 12 and a four dimensional phase converter circuit [13].

We now turn to the important question of the rate of flow of information about other variables into the given variable, i.e. the variable whose time series is known, say $x_{1}$. To investigate this question we start with the initial value $x_{1}(0)$ at $t=0$. This alone does not tell us anything about the other variables. As time evolves information about other variables flows into $x_{1}$ and the method presented above allows us to extract this information. As a specific example consider the Rössler system with time series for $x_{1}$. Fig. 2 shows the plot of the asymptotic error $e_{2}$ of the estimation of $x_{2}(0)$ for large $n$, denoted by $e_{\infty}$, as a function of the total time interval $\tau=(d-1) \Delta t$ for which the time series of $x_{1}$ is used in the calculations. We observe that the accuracy of estimation improves with $\tau$ and shows a power law behaviour of the type

$$
e_{\infty}=\tau^{-\nu}
$$

Fig. 2 shows a very good straight line behaviour on a loglog plot over seven orders of magnitude and the slope gives the value of the exponent $\nu=2.00 \pm 0.01$. The power law dependence of the accuracy on time interval as in Eq. (12) shows that the information entropy in terms of bits or partitions grows logarithmically with time. Note that this flow of information is different from the usual linear growth of information entropy with time in chaotic systems which corresponds to an exponential dependence of $e_{\infty}$ on $\tau$. The excellent straight line fit over seven orders of magnitude that we observe in Fig. 2, makes us believe that the power law dependence of Eq. (12) and the corresponding logarithmic growth of information content with time is a genuine property of dynamical systems. Further, the exponent $\nu$ appears to be universal and has the same value within numerical errors for the various examples that we have considered.

Now we will discuss an important application of our method for secure communications. Let us suppose that Eq. (1) describes a $d$-dimensional chaotic system at the transmitter end and a replica of the same at the receiver end. Let $s(i), i=1,2, \ldots, k$ be the information signal to be encoded for communication and without loss of generality let $x_{1}(t)$ be the scalar output signal to be transmitted. The signal $s(t)$ is encoded as follows. While choosing the initial conditions of the vector $\mathbf{x}(0)$, the component $x_{1}(0)$ is chosen arbitrarily while the other $d-1$ components are chosen as $x_{i}(0)=s(i-1), i=2, \ldots, d$. Using the initial vector, $\mathbf{x}(0)$, we evolve Eq. (11) for $d-1$ time steps of $d t$ to obtain the first $d$ values of the scalar variable $x_{1}$. This is the first stage of evolution. The vector $\mathbf{x}\left(t_{1}\right)$, where $t_{n}=n(d-1) d t$, is modified using $s(i)$ as explained below (Eq. (13)) and the resultant is evolved for $d-1$ time steps completing the second stage. The process is repeated as often as required. After completion of each stage the vector $\mathbf{x}\left(\mathbf{t}_{\mathbf{n}}\right)$ is modified as follows.

$$
\begin{aligned}
& x_{1}\left(t_{n}\right)=x_{1}\left(t_{n}\right) \\
& x_{i}\left(t_{n}\right)=x_{i}\left(t_{n}\right)+s(n(d-1)+i-1), \quad i=2, \ldots, d .
\end{aligned}
$$

Thus, assuming that $k$, the number of elements of information signal $s(i)$, is divisible by $d-1$ we get $k+1$ values of the encoded signal $x_{1}$.

The recovery of the information signal at the receiver end using our procedure of initial condition estimation is straightforward. At each stage $d-1$ values of $x_{1}$ unable us to estimate the initial conditions thereby recovering the signal from Eq. (13).

The communication procedure described above has several advantages. Note that (a) The transmitted signal $x_{1}$ is not directly modulated by the information signal $s$. (b) The information signal is added to more than one variables. Due to these reasons, local approximation for the flow of the dynamical system in the embedded phase space [14] cannot be used to attack the coding procedure. Secondly, the transmitted signal is of the same size as that of the information signal, and there is no initial transient as required in other synchronization based methods [3]. Thirdly, as in other methods using chaotic encoding the same signal $s$ will give different outputs for reruns of the coding procedure thereby making any attack very difficult.

Another important application of our initial condition estimation method is in the problem of synchronization of two identical chaotic systems coupled unidirectionally by a scalar signal. The knowledge of initial conditions enables us to obtain immediate synchronization thereby eliminating transients [5]. Also, the method could be 
used repeatedly to maintain synchronization. This will be particularly useful for systems where the largest conditional Lyapunov exponent is positive and other methods of synchronization fail [5].

To summarize, we have introduced a novel yet simple method to estimate initial conditions of a multivariable dynamical system from a given scalar signal. Our method is based on a multidimensional Newton-Raphson method where we include the time evolution of the system. The method gives a reasonably fast convergence to the correct initial state. The required length of the time series is very small. The method works even when the largest conditional Lyapunov exponent is positive. We find that the information flow between different variables grows logarithmically with time. This is different from the usual linear growth of information in chaotic systems. An important application of the method is in secure communications. This method of communication has several advantages as compared to other methods of communication based on nonlinear dynamical systems.

[1] R.L. Devaney, An Introduction to Chaotic Dynamical Systems, (The Benjamin / Cummings Pub. Co. Inc., Menlo
Park - California, 1986).

[2] W.H. Press, S.A. Teukolsky, W.T. Vetherling and B.P. Flannery, Numerical Recipes in C, 2nd ed. (Cambridge University, New York, 1992), p. 379.

[3] K.M. Cuomo and A.V. Oppenheim, Phys. Rev. Lett. 71, 65 (1993); K. John and R.E. Amritkar, Int. J. Bifurcation Chaos 4, 1687 (1994); U. Parlitz, L. Kocarev, T. Stojanovski, H. Preckel, Phys. Rev. E 53, 4351 (1996) and references therein.

[4] S. Hayes, C. Grebogi and E. Ott, Phys. Rev. Lett. 70, 3031 (1993).

[5] L.M. Pecora and T.L. Carroll, Phys. Rev. Lett. 64, 821 (1990).

[6] E. T. Whittaker A treatise on the analytical dynamics of particles and rigid bodies, $4^{\text {th }} \mathrm{Ed}$. (Dover publications, NY, 1944) p. 268.

[7] O.E. Rössler, Phys. Lett. A 57, 397 (1976).

[8] E.N. Lorenz, J. Atmos. Sci. 20, 130 (1963).

[9] L.O. Chua, L. Kocarev, K. Eckart and M. Itoh, Int. J. Bifurc. and Chaos 2, 705 (1992); also see Ref. [6] p.238.

[10] T. Rikitake, Proc. Cambridge Philos. Soc. 54, 89 (1958).

[11] C. Flynn and N. Wilson, Am. J. Phys. 66(8), 730 (1998).

[12] J.M. Wersinger, J.M. Finn, E. Ott, Phys. Rev. Lett. 44, 453 (1980).

[13] T. Yoshinaga and H. Kawakami in Nonlinear dynamics in circuits, ed. L.M. Peccora and T.L. Carroll (World Scientific Pub. Co. Pte. Ltd., Singapore, 1995) p. 111.

[14] H.D.I. Abarbanel, R. Brown, J.J. Sidorowich, L.Sh. Tsimring, Rev. of Mod. Phys. 65, 1331 (1993).

FIG. 1. Plot (a) shows the errors $e_{2}$ and $e_{3}$ on a logarithmic scale, as a function of $n$, the number of iterations of our method for Rössler system when time series for variable $x_{1}$ is given. The pluses show the values for $e_{2}$ and the crosses those for $e_{3}$. Errors are seen to approach zero as $n$ increases. The parameters are $(a, b, c)=(0.2,0.2,9.0)$, the time step $\Delta t=0.01$ and we use fourth order Runge-Kutta method for the time evolution of Rössler equations. (We have also checked our results with fifth and sixth order Runge-Kutta methods.) Similarly plot (b) shows the errors $e_{1}$ (pluses), and $e_{3}$ (crosses) as a function of $n$ when time series for $x_{2}$ is given. Plot (c) shows the errors $e_{\mid}$(pluses), and $e_{2}$ (crosses) as a function of $n$ when time series for $x_{3}$ is given.

FIG. 2. The points in the graph show the asymptotic error $e_{\infty}$ of initial state estimation as a function of $\Delta t$. The case under consideration is the same as in Fig. 1(a) for $e_{2}$ averaged over several realizations. A good straight line fit is obtained over seven orders of magnitude and has a slope of $-2.0 \pm 0.01$. This indicates a logarithmic flow of information from variable $x_{2}$ to variable $x_{1}$. 

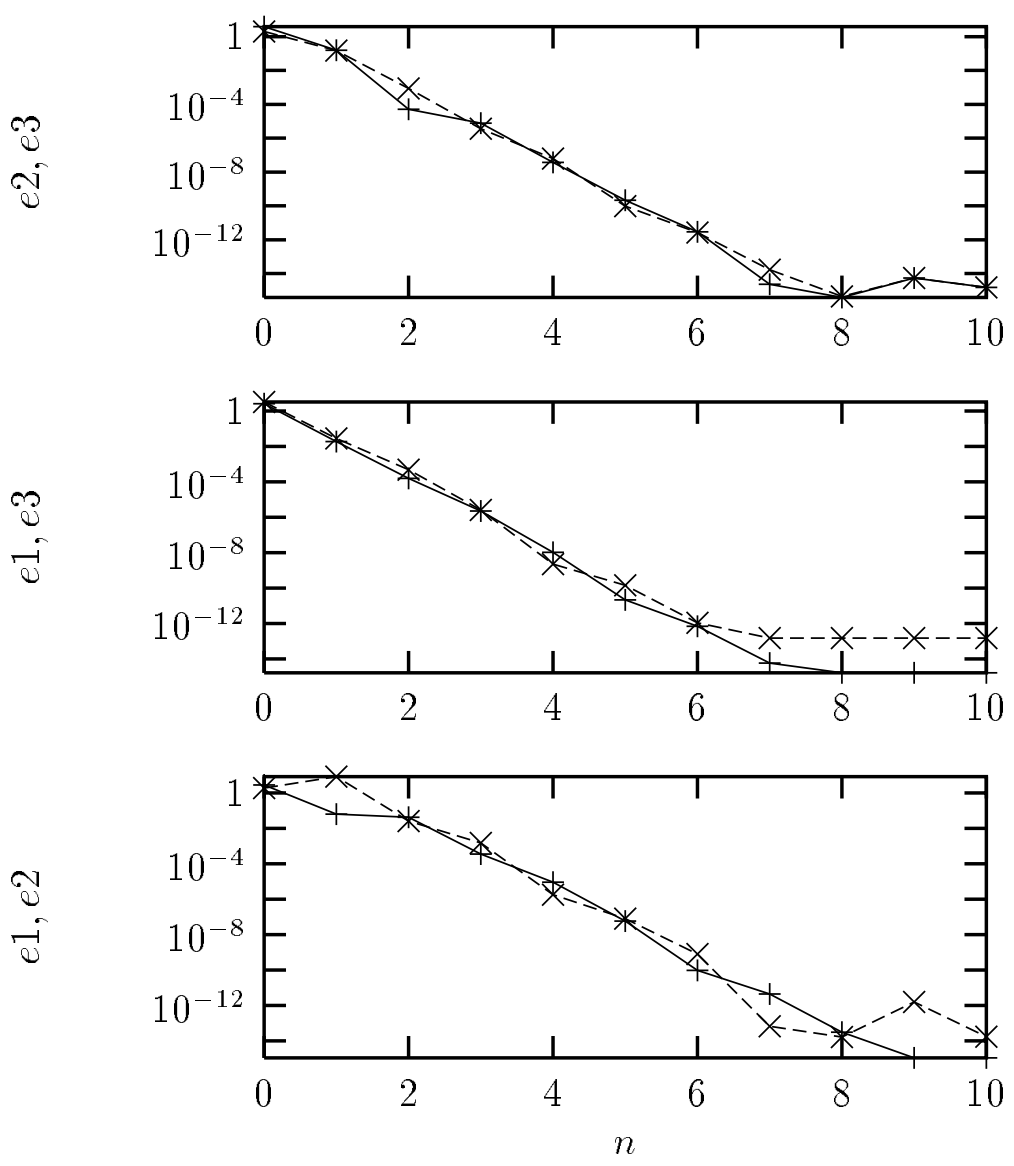

Fig. 1

(AM\&REA \& \&R\&) 


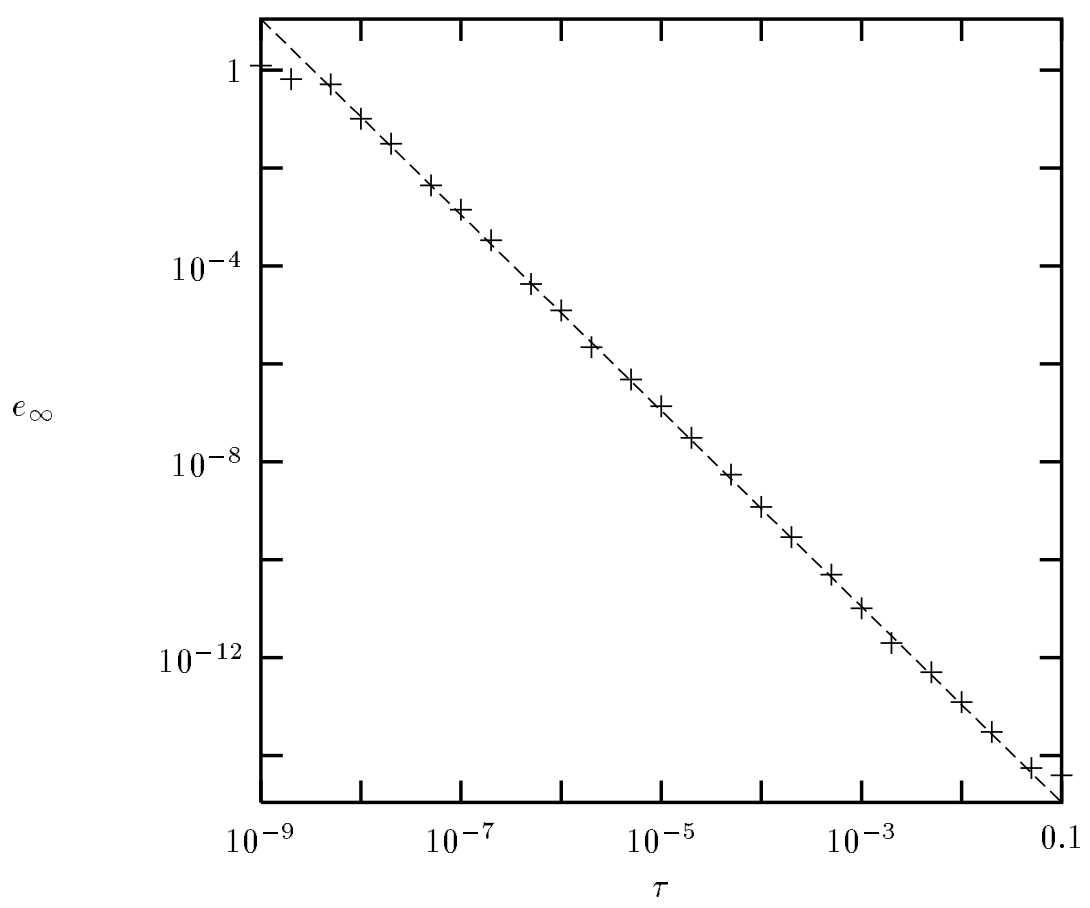

Fig. 2

(AM, REA \& DRK) 
